\title{
Philosophiques
}

\section{La place de l'éviction et de la fuite dans le perfectionnement éthique}

\section{Rose Goetz}

Volume 29, numéro 1, printemps 2002

Spinoza sous le prisme de son anthropologie

URI : https://id.erudit.org/iderudit/009565ar

DOI : https://doi.org/10.7202/009565ar

Aller au sommaire du numéro

Éditeur(s)

Société de philosophie du Québec

ISSN

0316-2923 (imprimé)

1492-1391 (numérique)

Découvrir la revue

Citer cet article

Goetz, R. (2002). La place de l'éviction et de la fuite dans le perfectionnement éthique. Philosophiques, 29(1), 89-98. https://doi.org/10.7202/009565ar d'utilisation que vous pouvez consulter en ligne.

https://apropos.erudit.org/fr/usagers/politique-dutilisation/ 


\title{
La place de l'éviction et de la fuite dans le perfectionnement éthique
}

\author{
ROSE GOETZ \\ Université Nancy II
}

\begin{abstract}
RÉSUMÉ. - À la proposition 69 d'Éthique IV, Spinoza affirme que l'homme libre, face aux dangers, fait montre d'une égale vertu quand il les fuit ou quand il triomphe. Mais, au cours de l'itinéraire éthique qui mène de la servitude à la liberté, interviennent de multiples formes de lutte, d'éviction et d'esquive, dont il s'agit d'apprécier l'efficacité libératrice à l'égard des « périls communs de la vie ».
\end{abstract}

\begin{abstract}
In Proposition 69 of Ethics IV, Spinoza claims that the free man, in the face of dangers, shows as much virtue when he flees from them as when he triumphs over them. But in the course of the ethical passage leading from bondage to freedom, a multiplicity of forms of struggle, eviction, and dodging occur, the liberating efficiency of which it is important to appreciate in regard to the "common dangers of life".
\end{abstract}

Au terme de la $4^{\mathrm{e}}$ partie de l'Éthique, quand surgissent en pleine lumière les contrastes entre la vie d'un homme conduit par l'affect ou par l'opinion et celle d'un homme conduit par la raison, Spinoza présente quelques observations concernant l'ingenium et la vivendi ratio de ce dernier. Entre autres traits, il note, à la proposition 69 , que " la vertu de l'homme libre se révèle aussi grande à décliner les dangers qu'à les surmonter ». La démonstration de la proposition repose sur ce qu'a établi la proposition 7 : « un affect ne peut être réduit ni ôté que par un affect contraire et plus fort ». Or l'audace et la crainte sont des affects qu'on peut concevoir de force égale, suscités avec une même intensité par la violence des puissances extérieures s'opposant à notre propre puissance. Il faut donc à l'âme autant de vertu ou de force pour réduire l'audace que pour réduire la crainte. La "force d'âme " (fortitudo), et la "fermeté d'âme" (animositas) invoquée dans le corollaire, doivent être entendues au sens que leur a donné Spinoza au scolie de la proposition 59 de la $3^{\mathrm{e}}$ partie. Relèvent de la fortitudo " toutes les actions qui suivent des affects se rapportant à l'âme en tant qu'elle comprend ». Partie constitutive de la fortitudo (avec la generositas qui n'entre pas ici directement en jeu), l'animositas est « le désir par lequel chacun s'efforce de conserver son être en vertu du seul commandement de la raison ". C'est parce que l'animositas est un désir qu'elle peut se mesurer à d'autres affects, s'immiscer dans leurs conflits. C'est parce que ce désir actif est affectivement plus fort que les affects passifs qu'il parvient à les ôter ou à les réduire. La proposition 14 de la $4^{\mathrm{e}}$ partie a fermement précisé que « la connaissance vraie du bon et du mauvais ne peut, en tant que vraie, réduire aucun affect, mais seulement en tant qu'elle est considérée comme un affect ». Par cet affect s'intensifie la puissance de l'âme, c'est- 
à-dire sa vertu. Ainsi l'homme conduit par la raison oppose-t-il victorieusement sa force propre à la force de l'audace et à celle de la crainte. D'où il suit, et tel est le corollaire de la proposition 69, qu'en présence des dangers, et selon les circonstances, c'est avec la même fermeté d'âme qu'il choisit la fuite ou le combat. Dans les deux cas, l'enjeu est identique : la conservation du conatus sous la dictée de la raison.

En dépit de sa rigueur démonstrative, ce texte éveille dans l'esprit du lecteur une double interrogation. Le frappe d'abord la discordance des expressions employées par Spinoza pour qualifier le combat des dangers par l'homme libre. Dans l'énoncé de la proposition, sa vertu se signale au fait qu'il les « surmonte ", qu'il en " triomphe ", qu'il en "vient à bout " (selon les traductions proposées de in superandis periculis). Dans la conclusion de la démonstration, elle consiste en ce qu'il « tente de les surmonter » : pericula superare tentat. Le soupçon s'insinue que la force d'âme ne mène pas infailliblement au succès ; en tout cas qu'elle réside déjà dans l'affrontement des dangers avant même leur destruction. Mais, s'il en était ainsi, Spinoza, dans le corollaire, aurait placé symétriquement à la fuite opportune, non le combat mais le combat opportun. S'il ne le fait pas, c'est que, à propos d'un homme libre, parler de « combat opportun » est un pléonasme. Le scolie de la proposition 10 de la $5^{\mathrm{e}}$ partie expliquera que, tant que nous ne connaissons pas parfaitement nos affects, nous devons concevoir une règle de vie droite et, notamment, " penser, pour nous débarrasser de la crainte, à la fermeté d'âme ; dénombrer et imaginer souvent les périls communs de la vie et comment on peut le mieux les éviter et les surmonter par la présence d'esprit et la force d'âme ". L'homme libre, tel qu'il est dépeint dans les dernières propositions de la $4^{\mathrm{e}}$ partie, a des idées adéquates de ses affects. Connaissant l'étendue et les bornes de sa puissance, il estime avec justesse les périls auxquels il est exposé. Si le rapport des forces lui est défavorable, il s'esquive. S'il engage le combat, l'issue n'en est pas douteuse. L'animositas qui l'induit à braver les dangers ne se distingue pas de celle qui l'en fait triompher. Mais qu'est-ce que cet " homme libre ", sinon la figure exemplaire du sage accédant au terme ultime du perfectionnement éthique ? En annonçant, au scolie de la proposition 73 , qu'il démontrera, dans la $5^{\mathrm{e}}$ partie, «jusqu'où » (quousque) la vertu humaine peut parvenir à atteindre une telle liberté, Spinoza en souligne l'idéalité. La substitution, dans la démonstration de la proposition 69, de pericula superare tentat à in superandis periculis, bien qu'elle n'exprime aucune modification de sens (ce qui ruinerait la preuve), n'a-t-elle pas pour effet de ramener l'attention, ne fût-ce que fugitivement, d'un idéal de perfection sur la voie réelle qui y conduit, de la liberté sur la libération au cours de laquelle l'âme s'affermit par degrés et réussit de mieux en mieux à surmonter les dangers ?

La réduction des attitudes de l' " homme libre » envers les dangers à la fuite opportune et au combat suscite une autre question. Le scolie de la proposition 69 définit le danger : " tout ce qui peut être cause de quelque mal, à savoir de tristesse, de haine, de discorde, etc. ». Or, dans des textes anté- 
rieurs, Spinoza a désigné, à maintes reprises, le mal, et le danger qui en est cause, comme les cibles d'actions diverses dont la destruction et la fuite ne sont que deux espèces parmi d'autres. Malum et periculum y paraissent comme les accusatifs des verbes destruere, amovere, removere, expellere, vitare, fugere, qui traduisent les multiples " stratégies du conatus ${ }^{1}$ envers ce qui l'affaiblit, ce qui le fait passer d'une plus grande à une moindre perfection. Serf ou libre, conduit par les affects ou par la raison, l'être humain, en tant que conatus, ne peut pas ne pas s'efforcer de supprimer les causes de sa tristesse tant qu'il n'est pas entièrement écrasé par elles. Certes, la raison seule et la fermeté d'âme qui en procède lui assurent la maîtrise des situations périlleuses par des moyens efficaces. Si ces moyens, chez l'homme libre, consistent dans la fuite opportune et dans le combat victorieux, c'est qu'il s'agit là des stratégies les meilleures. On peut imaginer, au pôle opposé, un homme tellement asservi par la crainte et l'audace qu'il oscillerait sans cesse entre la fuite aveugle et le combat téméraire, ne devant sa persévération dans l'existence qu'au concours fortuit des causes extérieures. Mais entre la réaction spontanée au danger et l'action rationnelle engagée contre lui, dans ce processus où la formation progressive d'idées adéquates dépend de la capacité du conatus à se libérer des affects passifs, ne faut-il pas prendre en compte toutes ses formes de lutte, d'éviction et d'esquive?

Spinoza ne présente pas ces formes isolément. Il les apparie souvent, sur le mode de l'association ou de la disjonction. Ainsi en va-t-il pour le couple amovere - destruere. Il arrive qu'amovere, "écarter ", soit synonyme de vitare, "éviter ". Mais l'acte d'écarter est préférentiellement lié à celui de détruire. Ayant posé, dans le corollaire de la proposition 13 de la $3^{\mathrm{e}}$ partie de l'Éthique, que "l'âme a en aversion d'imaginer ce qui diminue ou contrarie sa puissance d'agir et celle du corps ", Spinoza énonce, dans le scolie, que " celui qui hait s'efforce d'écarter et de détruire la chose qu'il a en haine ". Le désir d'anéantir prolonge celui de repousser comme, dans le cas inverse et symétrique de l'amour, le désir de conserver la chose aimée prolonge le désir de l'avoir présente. La proposition 28 semble les dissocier en substituant vel à $e t$ : « tout ce qui mène à la tristesse, nous nous efforçons de l'écarter ou de le détruire ». Mais sa démonstration, qui s'appuie sur la proposition 13, précise vel par sive : " nous nous efforcerons de le détruire, c'est-à-dire de l'écarter de nous... » Qu'il ne s'agisse pas d'une véritable alternative est confirmé par la démonstration de la proposition 39 où " écarter ou détruire " prouve que « qui a quelqu'un en haine s'efforcera de lui faire du mal ». Si tout l'effort de l'homme affecté de tristesse tend, selon la proposition 37, à écarter la tristesse, sa puissance d'agir, proportionnelle à la force de l'affect, s'intensifie dans la destruction des causes de la tristesse. La joie que nous éprouvons à imaginer disparu ce que nous haïssons secourt notre effort pour le détruire.

1. On peut utiliser au pluriel l'excellente expression de Laurent Bove. Voir Bove, Laurent, La stratégie du conatus, Paris, Vrin, 1996. 
C'est l'affect de joie qui permet d'amovere les causes de la tristesse enveloppée dans la haine.

Une joie plus grande, une augmentation de la puissance d'agir, se produit quand amovere n'implique plus destruere. On le voit, à la proposition 44, à propos de "la haine, entièrement vaincue par l'amour, qui se change en amour ». Dans cette métamorphose, deux joies confluent : la joie de l'amour en tant qu'amour, la joie née de ce que l'effort d'écarter la tristesse enveloppée dans la haine est " tout à fait aidé ", puisque l'idée de celui que l'on haïssait accompagne cette joie comme cause. C'est pourquoi cet amour est plus fort que si la haine ne l'avait pas précédé. Mais à ce stade où l'âme n'a que des idées confuses, l'amour se mue en haine aussi facilement que la haine en amour. L'imagination inverse le rapport de cause à effet que Spinoza a énoncé au scolie de la proposition 9 : " Nous ne nous efforçons vers aucune chose, ne la voulons, appétons ni désirons, parce que nous la jugeons bonne ; mais, au contraire, nous jugeons qu'une chose est bonne parce que nous nous efforçons vers elle, la voulons, appétons et désirons ». Est bon ce qui nous rend joyeux ; mauvais, ce qui nous attriste. L'imagination attribue à des choses extérieures le pouvoir de susciter l'augmentation ou la diminution de la puissance d'agir du conatus tandis que, en réalité, le conatus constitue ces choses en aimables ou haïssables selon les rencontres fortuites de notre corps avec elles. Dans cet état d'assujettissement à des causes extérieures, il n'est pas étonnant que l'âme passe aisément de l'amour à la haine et puisse éprouver, comme cela se produit dans la jalousie, l'un et l'autre en même temps. Seule l'animositas de l'homme dirigé par la raison exclut ces fluctuations. "Un homme à l'âme forte n'a personne en haine ", "la haine doit être vaincue par l'amour ", dit Spinoza au scolie de la proposition 73 de la $4^{\mathrm{e}}$ partie. Des idées adéquates découlent les affects actifs dont l'homme libéré est la cause adéquate. Mais la connaissance par notions communes, qui permet à l'âme de former des idées adéquates, a pour point de départ les idées inadéquates qu'elle a d'elle-même, de son corps et des corps extérieurs. Pour arriver à " considérer à la fois plusieurs choses, connaître les conformités qui sont entre elles, leurs différences et leurs oppositions ", selon les termes du scolie de la proposition 29 de la $2^{\mathrm{e}}$ partie de l'Éthique, elle doit découvrir ce qui est commun à son corps et aux autres corps. L'expérience répétée d'affects joyeux, quoiqu'encore passifs, mène l'âme au discernement de ce qui convient au corps. Elle est une étape indispensable à l'apparition d'affects actifs, nécessairement joyeux, nés de la raison. La joie d'écarter la tristesse en détruisant sa cause supposée, la joie, moins passive, de l'écarter en transformant cette cause en cause de joie, contribuent à la conquête de la joie active qui "se rapporte à l'âme en tant qu'elle comprend ". Tristitiam amovere est un impératif constant de tous les stades du perfectionnement éthique. Pour ce faire, l'homme libre est mieux armé que l'homme asservi. Mais en tant que mode fini, son conatus est exposé à une multitude de périls auxquels il fait face diversement. C'est ce qu'affirme le chapitre 8 de l'Appendice qui clôt la $4^{\mathrm{e}}$ partie : "Tout ce qui est donné dans la nature des 
choses que nous jugeons être mauvais, c'est-à-dire pouvoir nous empêcher d'exister et de jouir d'une vie rationnelle, il nous est permis de l'écarter par la voie paraissant la plus sûre " (" écarter » traduit ici removere).

Parmi les réactions spontanées du conatus au danger, à côté de l'éviction, et parfois lié à elle, se trouve l'évitement que Spinoza associe à ce qui en apparaît la condition ou le moyen : l'abstention. Vitare et Abstinere forment couple. Dans la démonstration de la proposition 39 de la $3^{\mathrm{e}}$ partie, qui, on l'a vu, porte sur l'effort d'écarter ou de détruire celui que l'on hait, Spinoza note que cet effort est réduit dans un cas bien précis. Si celui qui éprouve de la haine « craint par là quelque chose de plus triste, ou (c'est la même chose) un plus grand mal pour lui, et s'il croit pouvoir l'éviter en ne faisant pas à celui qu'il hait le mal qu'il méditait, il désirera s'abstenir de lui faire du mal ; et cela, d'un effort plus grand que celui qui le portait à faire du mal et qui, donc, prévaudra ". Cet homme est mû par la peur (timor), définie dans le scolie comme une espèce de la crainte (metus) : " la crainte en tant qu'elle dispose l'homme à éviter un mal qu'il juge devoir venir par un mal moindre ». La peur est l'affect qui pousse le conatus à ne pas vouloir ce qu'il veut et à vouloir ce qu'il ne veut pas. Il arrive que le mal dont un homme s'effraie le frappe d'une stupeur telle qu'elle anéantit son désir de l'éviter. Cette épouvante, Spinoza la nomme « consternation » et la décrit, dans l'explication de la définition $47 \mathrm{de}$ l'Appendice, comme le produit d'une peur double. L'homme consterné, affolé, est paralysé à la fois par l'ampleur de son effroi et par sa fluctuation entre les tourments de deux dangers. Il ne sait lequel des deux détourner (avertare) et son désir d'écarter (amovere) le mal est réduit. Ici, vitare rejoint amovere : ne pas " éviter» vaut ne pas « éloigner».

Mais le plus souvent, vitare se rapporte au choix du moindre mal. Ainsi l'avare, évoqué dans l'explication de la définition 48, en dépit de son amour immodéré des richesses, jette-t-il ses richesses à la mer pour échapper à la mort. Et la mort elle-même peut être choisie comme un moindre mal par qui est menacé d'une mort pire. Le suicide de Sénèque, au scolie de la proposition 20 de la $4^{\mathrm{e}}$ partie, est un exemple, parmi d'autres différents, illustrant la thèse selon laquelle on ne se donne jamais la mort que sous la contrainte de causes extérieures : « ... ou encore on est, comme Sénèque, forcé par l'ordre d'un tyran de s'ouvrir les veines, c'est-à-dire qu'on désire éviter un mal plus grand par un moindre ». Hormis ces cas de défaite totale du conatus par des causes extérieures à sa nature et qui lui sont contraires, personne n'omet de chercher ce qui lui est utile, c'est-à-dire de conserver son être. Or, le scolie de la proposition 18 a montré que "rien n'est plus utile à l'homme que l'homme". Selon la proposition 73, «l'homme qui est dirigé par la raison est plus libre dans la Cité, où il vit selon le décret commun, que dans la solitude où il n'obéit qu'à lui-même ». Si peu d'hommes vivent sous la conduite de la raison, ceux que dirigent seulement leurs affects reconnaissent déjà les avantages de la société et proclament l'homme "dieu pour l'homme». Ils éprouvent, dit Spinoza au scolie de la proposition 35, " qu'ils peuvent beau- 
coup plus aisément se procurer par une aide mutuelle ce dont ils ont besoin, et qu'ils ne peuvent éviter les périls les menaçant de partout qu'en joignant leurs forces ». Pour esquiver les dangers omniprésents auxquels il est exposé, le conatus doit s'abstenir de tout repli sur lui-même. Dans ce scolie, nous rencontrons les trois types d'ennemis mortels de la nature humaine, qui sont aussi les pires adversaires d'une éthique conçue à la manière spinoziste : les Satiriques qui tournent en dérision les choses humaines, les Théologiens qui les détestent et les Mélancoliques qui les méprisent. Or, même s'il n'obéit aux lois de la cité que par crainte de sanctions répressives, le citoyen y a plus de puissance que l'individu solitaire. Sa crainte est une tristesse. Mais l'augmentation de sa puissance est une joie. Une accumulation de joies dues à l'association peut le mener, dans la cité qui " tient lieu de raison à ceux qui n'en ont pas ", selon la formule de Deleuze, à la formation de sa propre raison dont la cité devient le lieu d'éclosion ${ }^{2}$. Entre la joie minimale d'éviter la mort, comme l'est, par exemple, celle d'une " multitude conquise en vertu du droit de guerre », dont il est question au $\$ 6$ du Traité Politique, et la joie d'éviter les périls ordinaires de la vie, que procure à ses membres un État où règnent concorde, paix et sécurité, toute une hiérarchie de passages d'un mal plus grand à un mal moindre doit prendre place. C'est dans la bonne cité, dont la virtus est la sécurité, que l'individu a des chances d'accéder à sa propre virtus, qui est liberté et force intérieure. Ce " saut » s'opère quand sa puissance de comprendre parvient à transformer la joie passive en joie active. La compréhension des raisons pour lesquelles certains dangers sont inesquivables contribue elle-même à cette joie. Au dernier chapitre de l'Appendice de la $4^{\mathrm{e}}$ partie de l'Éthique, Spinoza écrit :

Nous supporterons d'une âme égale les événements contraires à ce qu'exige la règle de notre utilité, si nous avons conscience de nous être acquittés de notre office, savons que notre puissance ne s'étendait pas jusqu'à nous permettre de les éviter, et que nous sommes une partie de la nature entière dont nous suivons l'ordre. Si nous comprenons cela clairement et distinctement, cette partie de nous qui se définit par l'intelligence, c'est-à-dire la meilleure, y trouvera un plein contentement et s'efforcera de persévérer dans ce contentement.

" Nous acquitter de notre office ", c'est poursuivre le bien. Par un désir qui tire son origine de la raison, le corollaire de la proposition 63 l'a précisé, " nous poursuivons le bien directement et fuyons le mal indirectement ». Un désir actif et joyeux procède de la connaissance du bien, non de celle du mal qui est la tristesse même en tant que nous en sommes conscients, et donc une connaissance inadéquate. C'est en quoi la fuite calculée de l'homme libre ne ressemble en rien à celle du peureux.

2. Deleuze, Gilles, Spinoza et le problème de l'expression, Paris, Éditions de Minuit, 1968, p. 247. 
Au terme de l'itinéraire de libération, fugere et destruere manifestent une égale activité. Au cours de la transformation des affects les plus passifs en affects moins passifs et susceptibles de devenir des affects actifs, vitare peut équivaloir à amovere ou removere. Cependant, le scolie du second corollaire de la proposition 45 invite à accorder une supériorité aux gestes de repoussement et d'expulsion. Spinoza y signale la nécessité impérieuse de chasser la mélancolie : melancholiam expellere. L'enjeu n'en est rien de moins que la conservation de soi : " chasser la mélancolie » est de même importance qu' « apaiser la faim et la soif ». Au scolie de la proposition 20, l'aversion pour la nourriture était associée au suicide et, comme lui, attribuée à la contrainte de causes extérieures. Anorexie et mélancolie mènent inéluctablement à la mort : ce à quoi aucun conatus ne peut, par définition, aspirer. La proposition 42 a établi que la mélancolie est toujours mauvaise. À la différence de la douleur, tristesse qui se produit quand une partie du corps est lésée, la mélancolie survient quand toutes les parties du corps sont également affectées. À cet effondrement général, il n'est d'issue que catastrophique. D'où l'urgence vitale de le bannir. Les moyens de l'éliminer préconisés par Spinoza dans le scolie cité, pour l'enchantement de tant de lecteurs surpris d'en trouver le recensement sous sa plume, sont les plaisirs variés de l'existence humaine :

Il est d'un homme sage de se refaire et recréer, en mangeant et buvant modérément de bonnes choses, ainsi qu'en usant des parfums, de l'agrément des plantes verdoyantes, de la parure, de la musique, des jeux qui exercent le corps, des spectacles, et d'autres choses du même genre dont chacun peut user sans dommage pour autrui.

Si cette façon de vivre s'accorde bien avec " la pratique commune ", elle se fonde sur la conception spinoziste du corps humain composé d'une multitude de parties hétérogènes qui requièrent continuellement une alimentation nouvelle et diversifiée. Lorsqu'il la reçoit, il est apte à remplir toutes ses fonctions et l'âme, idée de ce corps, devient apte à comprendre beaucoup de choses à la fois. Qu'il faille, dans l'usage des plaisirs, se garder de tout excès ne découle pas d'un impératif moral de modération, mais du constat que l'excès déséquilibre la puissance du corps à être affecté en toutes ses parties. L'excès conduit au dégoût, à la nausée qui n'est plus plaisir mais tristesse. Le plaisir doit être pure joie, car « plus grande est la joie dont nous sommes affectés, plus grande la perfection à laquelle nous passons, plus il est nécessaire que nous participions de la nature divine ". Dans ce texte de la $4^{\mathrm{e}}$ partie de l'Éthi$q u e$, inséré dans une étude comparée des affects selon leur utilité ou leur nocivité, on a là une sorte d'anticipation-éclair de la $5^{\mathrm{e}}$ partie où tout affect joyeux sera rapporté à Dieu et où l'acquiescentia in se ipso s'épanouit en béatitude.

Il n'est donc pas étonnant que l'on retrouve dans ce scolie l'ennemie à abattre : « la farouche et triste superstition » qui prohibe le plaisir. Spinoza y dénonce l'« envieux " qui se réjouit du malheur des autres et tient pour vertu les larmes, les sanglots, la crainte et les autres marques d'impuissance d'une 
âme. L'enjeu est bien d'ordre théologique. Car ce sont les "Théologiens ", prédicateurs d'humilité et de mortifications, qui tentent de faire croire en une divinité envieuse, jouissant de nos peines et de nos faiblesses. Dans la Préface du Traité théologico-politique, Spinoza les dépeint coupables du travestissement en religion de la crainte que le régime monarchique impose aux hommes afin qu' "ils combattent pour leur servitude comme s'il s'agissait de leur salut ». Les orateurs des églises dégénérées en théâtres y sont présentés comme des charlatans mus, non par le désir d'instruire leurs naiffs auditeurs, mais par celui de les captiver en les stupéfiant. Comme la peur empêche l'homme de chercher les vrais moyens de son salut, la fascination par les fictions extravagantes de la superstition l'empêche d'exercer sa raison. Les ravages effectifs de la superstition découlent de son interprétation délirante de la nature des choses. Spinoza semble toutefois entretenir l'espoir que la mélancolie qu'elle prétend instiller se retourne contre elle. Au scolie de la proposition 63 de la quatrième partie de l'Éthique, il affirme :

Les superstitieux qui savent réprouver les vices plutôt qu'enseigner les vertus et qui, cherchant non à conduire les hommes par la raison mais à les contenir par la crainte en sorte qu'ils fuient le mal plus qu'ils n'aiment les vertus, ne tendent à rien d'autre qu'à rendre les autres aussi misérables qu'eux-mêmes. Il n'est donc pas étonnant qu'ils soient le plus souvent insupportables et odieux aux hommes.

Selon la proposition 63, celui qui fait le bien pour éviter le mal n'est pas conduit par la raison (vitare dans l'énoncé de la proposition et fugere dans le scolie sont synonymes), puisque tous les affects qui se rapportent à la raison sont des affects de joie et de désir. Le chapitre XIII de l'Appendice soulignera à nouveau que ceux qui censurent les hommes et flétrissent leurs vices au lieu d'enseigner les vertus, qui s'emploient "à briser les âmes au lieu de les fortifier ", sont insupportables aux autres, en ajoutant qu'ils le sont aussi à eux-mêmes. Beaucoup de ces mélancoliques, égarés par un faux zèle religieux, ont choisi de vivre parmi les bêtes plutôt qu'avec leurs semblables. Spinoza les compare à ces adolescents qui fuient (confugiunt) dans le service militaire les remontrances de leurs parents et préfèrent les inconvénients de la guerre et l'autorité d'un tyran aux douceurs domestiques et aux réprimandes paternelles.

Dans l'éthique spinoziste, la mélancolie est frappée d'un décret d'expulsion. On ne peut ni la fuir, ni s'y dérober, ni la détourner, ni s'y soustraire. On ne peut que la combattre sans merci, puisqu'il y va du conatus lui-même en tant qu'effort pour persévérer dans son être. On peut aussi la prévenir. Thérapeutiques ou prophylactiques, les moyens de ce combat sont tous directs et positifs : il s'agit de fournir continûment aux diverses parties du corps les " aliments » dont il a besoin pour être, en son entier, apte à tout ce qui suit de sa nature. Ces nourritures sont les plaisirs exclusifs de toute tristesse : les joies pures qui, rapportées à l'âme, développent son aptitude à comprendre. Cer- 
tes, cette culture des plaisirs implique-t-elle des stratégies d'évitement, ne serait-ce que d'évitement des excès. Les affects « auxquels nous sommes quotidiennement en proie ", remarque Spinoza dans le scolie de la proposition 44 , se rapportent la plupart du temps à une partie du corps plus que les autres affectée. L'âme est ainsi absorbée dans la considération d'un seul objet et ne peut penser à rien d'autre. Cela peut aller jusqu'au délire, comme, par exemple, chez ceux qui brûlent d'amour et ne font que rêver, nuit et jour, de la femme aimée. Une vigilance doit donc s'exercer à l'égard de cette domination du corps par une seule affection ou par quelques-unes seulement. L'usage de tous les plaisirs est la condition d'une aptitude de l'âme à comprendre le plus de choses possibles, à former le plus grand nombre possible d'idées adéquates. Mais si nous devons fuir ou éviter les objets qui requièrent une attention exclusive, les détourner ou nous en détourner, c'est bien pour briser les limites dans lesquelles ils nous emprisonnent et libérer la capacité d'agir de notre corps, la capacité de comprendre de notre âme.

Dans le scolie de la proposition 10 de la $5^{\mathrm{e}}$ partie, Spinoza indique à l'homme en voie de libération comment se servir du « pouvoir d'ordonner et d'enchaîner correctement les affections du corps en sorte de n'être pas facilement affecté par des affects mauvais ». Il faut, notamment, "prêter attention à ce qu'il y a de bon en chaque chose afin que ce soit toujours un affect de joie qui nous détermine à agir ». Ainsi, si quelqu'un s'aperçoit qu'il recherche trop la gloire, il pensera au bon usage qu'on en peut faire, à la fin en vue de laquelle on la poursuit et aux moyens de l'acquérir, non à ses abus, à sa vanité ou à l'inconstance des hommes dont dépend sa reconnaissance. Chercher le bon en toute chose, c'est éviter indirectement le mauvais sans jamais penser à ce mauvais. Pour ce motif encore, sont condamnés ceux qui se complaisent à contempler les vices des hommes et à dénigrer la nature humaine, alors que "qui s'emploie à maîtriser ses affects et ses appétits par le seul amour de la liberté s'efforcera, autant qu'il peut, de connaître les vertus et leurs causes et de s'emplir l'âme du contentement qui naît de leur connaissance vraie ». L'acquiescentia, qui n'est, au départ, qu'une joie née de la contemplation par l'homme de sa puissance d'agir, devient, au cours du processus de libération, « un contentement intérieur souverain né de la conduite droite de la vie ", comme le dit Spinoza dans ce scolie, puis, liée à la connaissance des essences singulières sub specie aeternitatis, béatitude.

Parmi tous les maux auxquels l'homme conduit par la raison ne pense jamais, figure le mal qui inspire à l'homme asservi par les affects tristes la plus effroyable des craintes : la mort. Cherchant uniquement le bon, la raison, qui poursuit en cela l'élan spontané du conatus, prescrit d'agir, de vivre, de conserver son être par la recherche de l'utile propre. Il n'est pas surprenant que, parmi les traits distinctifs de l'ingenium du sage dépeint à la fin de la $4^{\mathrm{e}}$ partie de l'Éthique, Spinoza relève son insouciance de la mort. Elle apparaît, à la proposition 67, comme le simple effet de la plénitude de ce qui occupe sa pensée, la vie : "Un homme libre ne pense à rien moins qu’à la mort, et sa sagesse 
est une méditation, non de la mort, mais de la vie ». C'est ce même homme libre qui choisit la fuite devant les dangers quand il ne peut les vaincre, parce qu'il a appris à en apprécier la gravité au cours de toutes les sortes de combats qu'il a engagés contre eux. Pour passer d'une fuite aveugle à une fuite opportune qui vaut une victoire, son conatus a dû s'aguerrir en de multiples expériences d'évitement et d'éviction des périls qu'il court comme toute chose singulière dans la nature. 